\title{
Labyrinthe
}

17 | 2004 (1)

Jacques Rancière, l'indiscipliné

\section{Subjectivation politique et énonciation littéraire}

\section{Marie De Gandt}

\section{OpenEdition}

Journals

Édition électronique

URL : http://journals.openedition.org/labyrinthe/178

DOI : $10.4000 /$ labyrinthe. 178

ISSN : 1950-6031

Éditeur

Hermann

Édition imprimée

Date de publication : 15 avril 2004

Pagination : 87-96

Référence électronique

Marie De Gandt, «Subjectivation politique et énonciation littéraire », Labyrinthe [En ligne], 17 | 2004 (1), mis en ligne le 13 juin 2008, consulté le 30 avril 2019. URL : http://journals.openedition.org/ labyrinthe/178; DOI : 10.4000/labyrinthe.178 


\section{SUBJECTIVATION POLITIQUE ET ÉNONCIATION LITTÉRAIRE}

Marie DE GANDT

L'apport de Rancière au débat contemporain pourrait s'énoncer comme une redéfinition de la politique. Dans cette entreprise théorique, un point résiste particulièrement à la compréhension : le mode d'existence concrète du sujet politique. Une lecture d'influence peut permettre, sinon de résoudre la question, du moins d'en cerner l'inspiration, en jetant un éclairage oblique sur la pensée de Rancière.

Dans La Mésentente, le philosophe reprend la définition aristotélicienne de «l'animal politique » comme « celui qui a le logos». Ce mot ne désigne plus, pour Rancière, la raison aristotélicienne, mais à la fois la «part » et la « voix ». Aussi la politique se présente-t-elle comme l'énonciation d'un tort, la profération d'une revendication par ceux qui sont sans parts dans le décompte des voix.

Cette définition de la politique comme parole du sujet est partagée par d'autres philosophes de cette génération. Elle prend alors deux directions principales. D'un côté, des philosophes comme Badiou pensent le sujet dans une perspective marxiste et freudienne. Badiou écrit ainsi du sujet qu'il « est le concept clef d'où résulte que soient pensables la décision, l'éthique et la politique ${ }^{1}$. » La question du sujet offre l'occasion de s'éloigner de la pensée du «maître », Althusser, dont Badiou présente la politique comme «du subjectif sans sujet». À l'écart de cette première tendance, Rancière ne recourt pas plus aux constructions du marxisme qu'à celles de la psychanalyse. Le sujet politique s'inscrit pour lui non pas dans une organisation mais dans la parole. Il se rapproche alors d'une autre voie contemporaine, celle qui inscrit la pensée du sujet dans la filiation heideggérienne. Dans les Cahiers confrontation, Jean-Luc Nancy proposait pour thème de réflexion: «Qui vient après le sujet? » La grande majorité des contributions à ce numéro reprend l'interrogation à partir de la notion d'ap-

1. Théorie du sujet, Paris, Seuil, coll. «L'ordre philosophique », 1982, p. 295. 
pel chez Heidegger. Dans son article, Rancière refuse au contraire de s'inscrire dans une conception de la perte ontologique: « Maintenant, après... il y a le lieu de cette aventure: ce sujet qui dit sa vérité dans la division et trouve sa paix dans la liaison ${ }^{2}$. » Dès lors, où placer la pensée du sujet politique proposée par Rancière?

Dans sa volonté de se définir toujours contre, Rancière ne facilite pas le travail de son lecteur. Il est difficile de comprendre comment il assimile l'action politique à l'énonciation. Aussi Badiou a-t-il pu lui reprocher de proposer une « phénoménologie historiciste de l'occurrence égalitaire »:

Mais Rancière ne fait pas de politique. Si en revanche on fait de la philosophie, il est exigible que les catégories ontologiques utilisées soient explicites et qu'on argumente leur cohésion. Mais Rancière après tout ne fait pas non plus de philosophie ${ }^{3}$.

Ce baiser de Judas appelle quelques mises au point. On peut penser que Rancière tendrait la joue à l'accusation et reprendrait la critique à son compte: sa politique, c'est du roman! Cette boutade résume le lien fort qui rattache philosophie et littérature chez un penseur qui récuse les distinctions entre disciplines. Mais le lien est aussi plus profond et moins évident: Rancière construit le sujet politique sur un modèle implicite, celui de l'énonciation littéraire.

«La question qui nous intéresse ici est la suivante: quelle nécessité essentielle lie la position moderne de l'énonciation poétique et celle de la subjectivité politique?» $(C M, 17)$. Pour répondre à cette question, au lieu de suivre la pensée de Rancière, et de retracer le «partage du sensible », on peut partir d'une hypothèse extérieure aux déclarations du philosophe. Le sujet politique, point obscur dans la pensée de Rancière, s'éclaire lorsqu'on le rapproche des théories de la polyphonie.

\section{POLITIQUE ET POLYPHONIE}

Dans La Mésentente, Rancière présente la politique comme une mode de subjectivation lié à la parole littéraire:

2. «Après quoi ? ", Cahiers confrontation n 20, 1989, p. 196.

3. Alain Badiou, Abrégé de métapolitique, Paris, Seuil, coll. «L'ordre philosophique », 1998, p. 131. 


\section{Subjectivation politique et énonciation politque}

Le dialogisme de la politique tient de l'hétérologie littéraire, de ses énoncés dérobés et retournés à leurs auteurs, de ses jeux de la première et de la troisième personne, bien plus que de la situation supposée idéale du dialogue entre une première et une deuxième personne (Més., 90).

Cette comparaison entre politique et littérature se présente en termes vagues, puisque le verbe «tenir de » évoque à la fois un lien analogique et un héritage. Rancière y définit aussi la façon dont les formes de l'argumentation et celles de la poésie sont liées dans une même conception esthétique de la place des choses et des sujets. Aussi la parole de rupture serait-elle indistinctement littéraire ou politique: "L'égalité fait effet dans le corps social sous forme d'existences suspensives, qui peuvent s'appeler littérature ou prolétariat $[\ldots] »(A B$, 194). C'est l'équivalence « littérature ou prolétariat», chez Rancière, qui pose un problème. On peut penser que le prolétariat est considéré comme littérature, parce qu'il a une forme d'existence ni historique ni irréelle, mais liée à une capacité de parole qui rompt l'ordre régnant. La littérature offre-t-elle une simple analogie à la parole politique, le reflet de ses mythes fondateurs à chaque époque, ou est-elle le modèle implicite qui donne sa forme ou son socle théorique à une pensée du sujet qui ne dévoile pas ses implicites? Rancière présente l'unité qui lie politique et littérature comme une donnée initiale. Cette évidence de surface masque la place qu'occupent les processus littéraires dans la théorie de la politique proposée par Rancière.

Dans La Mésentente, les termes «dialogisme » et « hétérologie » invitent à une comparaison avec les théories de Bakhtine. Le dialogisme est chez Rancière le nom qu'il donne à une parole qui rompt le consensus, qui refuse les rôles du dialogue traditionnel, et qui prend des accents « carnavalesques » :

Ainsi peut-il y avoir des moments de la communauté, non pas ces moments festifs que l'on décrit parfois, mais des moments dialogiques $(A B, 174)$.

Et certains passages font entendre la terminologie bakhtinienne en échos diffus :

La logique de la subjectivation politique est ainsi une hétérologie, une logique de l'autre, selon trois déterminations de l'altérité. Premièrement, 
elle [...] est toujours en même temps le déni d'une identité imposée par un autre, fixée par la logique policière. [...] Deuxièmement, elle est une démonstration, et une démonstration suppose toujours un autre à qui elle s'adresse, même si cet autre refuse la conséquence. [...] Troisièmement, la logique de la subjectivation comporte toujours une identification impossible (Més., 89-90).

Serait-ce tordre le texte de Rancière que d'y lire la réécriture du topos bakhtinien d'une présence de l'autre dans la parole du sujet? Dans la citation de Rancière, l'autre se présente sous trois formes, celui auquel le sujet s'oppose, celui auquel il s'adresse, et celui auquel il s'identifie. Chacun de ces termes trouve son correspondant dans la théorie de la polyphonie littéraire.

Dans La Poétique de Dostoïevski, le dialogisme se définissait déjà comme ce qui rompt l'ordre policier. Pour Bakhtine, la littérature est le lieu où se fait entendre une humanité excédentaire:

Le postulat de la véritable prose romanesque, c'est la stratification interne du langage, la diversité des langages sociaux et la divergence des voix individuelles qui y résonnent $t^{4}$.

La dissension politique vient s'inscrire jusque dans le sujet: « De ce monde remis en question, inséparable d'une prise de conscience sociale plurivocale, le romancier parle dans un langage diversifié et intérieurement dialogisé ${ }^{5}$. P Pour Bakhtine, non seulement chaque mot proféré contient une contradiction «bivocale » mais le locuteur prévoit l'altérité de la réception dans la structure de son propos: il parle pour et contre autrui, cherchant à anticiper sa position adverse. La parole fait détour par l'altérité, ce qui empêche toute identification du sujet à son dire. De même, chez Rancière, le discours du sujet s'adosse à l'autre pour contester son autorité, pour appeler son attention, mais aussi pour en faire le détour d'une revendication qui ne soit pas identitaire: «Qu'est-ce qu'un processus de subjectivation? C'est la formation d'un un qui n'est pas un soi mais la relation d'un soi à un autre $»(A B, 118)$.

\footnotetext{
4. Esthétique et théorie du roman, Gallimard 1978, traduit par Daria Olivier, p. 90.

5. Ibid., p. 150.
} 
Le parallèle entre Bakhtine et Rancière trouve évidemment ses limites. Pour Bakhtine, la littérature polyphonique est une parole qui a été réprimée par la littérature officielle dont elle récusait l'autorité. On pourrait alors être tenté de rapprocher cette conception du roman de la parole politique chez Rancière. Or, ce serait méconnaître la nature du tort politique. En effet, la politique selon Rancière ne peut être ramenée à la dissension. La mésentente implique un désaccord beaucoup plus fondamental, de nature ontologique. L'inspiration bakhtinienne de Rancière ne s'inscrit donc pas dans le cadre d'une conception marxiste de la littérature. Mais la théorie de la polyphonie permet de comprendre le fonctionnement d'une parole qui ne serait pas une parole d'identité. Plus que la dissension et le dialogisme, c'est la désidentification qui fait le fond de la polyphonie. Bakhtine définit le sujet romanesque comme une voix qui ne s'appartient pas, qui ne prend pas corps ni identité. Rancière n'hérite-t-il pas de cette conception nouvelle du sujet lorsqu'il définit le sujet politique par la « désidentification »? «Les voies de la subjectivation politique ne sont pas celles de l'identification imaginaire mais de la « désincorporation » littéraire » $(P S, 64)$. Cette parole d'un sujet dépossédé de toute identité vient des théories de la polyphonie, non plus seulement celles de Bakhtine, mais celles des linguistes.

Reprenant à Bakhtine la théorie de l'énonciation romanesque, les linguistes y ont adjoint les découvertes de Benveniste sur la personne grammaticale. Rancière semble se faire l'écho de ces recherches linguistiques lorsqu'il présente la non-coïncidence à soi du sujet dans l'acte de parole:

Le moment propre de l'œuvre, c'est l'inscription d'un il entre ces deux je, d'une hétéronomie dans le rapport de l'un à l'autre. Si la littérature témoigne de quelque chose qui importe à la communauté, c'est par ce dispositif qui introduit l'hétéronomie dans le je $(A B, 193)$.

Plus que la polyphonie romanesque, c'est bien la polyphonie linguistique qui semble informer la pensée du sujet politique chez Rancière. La linguistique moderne a défini un sujet de parole qui serait potentiellement présent dans son propos sans y inscrire son identité. Elle offre alors à la pensée politique un modèle fort arrangeant: l'utopie d'un sujet de parole éternellement potentiel et jamais entièrement actualisé. 


\section{L'ACTE D'ÉNONCIATION ET L'ÉTERNEL POSSIBLE}

Rancière a accordé une grande attention aux formes linguistiques de l'énonciation littéraire, tout en se défendant d'une assimilation trop facile: «Selon moi, il n'y a pas de correspondance terme à terme entre les formes romanesques et les formes de l'action politique ${ }^{6}$. »Par ses dénégations, Rancière laisse informulée la dette littéraire de sa pensée politique. En étudiant les formes littéraires, il n'aurait fait qu'appliquer à des objets nouveaux l'analyse de la parole politique qu'il avait déjà pratiquée sur les «prolétaires ». Ainsi, il a relevé dans la poésie et le roman du $\mathrm{XIX}^{\mathrm{e}}$ et du début du $\mathrm{XX}^{\mathrm{e}}$ siècle, chez Wordsworth, Flaubert, Rimbaud ou Proust, les éléments d'une subjectivation complexe : émergence du style indirect libre, effacement du narrateur, division problématique entre l'énoncé et sa source. Rancière présente ces éléments comme les marques d'un régime de parole historique, mais on peut penser qu'ils ont perdu leur statut de preuve pour devenir un paradigme. Cette construction littéraire du sujet serait silencieusement passée dans les constructions théoriques, ou imaginaires, du philosophe, pour devenir le socle inquestionné de toute subjectivation politique. La pensée politique de Rancière reposerait en fait sur une conception du sujet de parole née dans les théories de l'énonciation littéraire. La dette envers l'énonciation littéraire est donc plus forte qu'un simple écho bakhtinien ne le laissait présager dans notre hypothèse de départ.

La linguistique semble avoir fourni les fondements inconscients de la pensée politique de Rancière. Interrogé par Diacritics, il déclarait s'éloigner du Linguistic Turn, dont il reconnaissait pourtant la portée politique: "S'ouvrait alors un nouvel espace, où les actes de parole étaient conçus comme des gestes politiques ${ }^{7}$. » Cette utopie de la linguistique des années 1960 fait entendre son influence dans la définition du sujet politique comme sujet de parole. La linguistique des années structuralistes a été l'espace de rencontre des philosophes et des littéraires. Il ne s'agissait pas alors, comme maintenant, de réduire la littérature à un choix sordide : être une expérience cognitive ou le reflet idéaliste d'une conception morale du monde. La littérature offrait le lieu

6. Entretien avec Substance, $\mathrm{n}^{\circ}$ 92, 2000.

7. «Dissenting Words», Diacritics vol. 30 n², 2000, p. 114. 


\section{Subjectivation politique et énonciation politque}

même où penser le sujet politique. Rancière aurait-il conservé cette conception de la littérature comme utopie? Sans reprendre l'articulation militante, il réutilise néanmoins les outils que la linguistique avait construits pour penser l'effectivité politique de la littérature.

Cette proximité dépasse la question du sujet d'énonciation. La politique de Rancière comporte un fond impensé qui s'éclaire lorsqu'on le rapproche de ses correspondants en linguistique et en théorie de la littérature. Ces deux disciplines ont évidemment déjà des partis pris idéologiques, qui peuvent expliquer la proximité théorique avec sa philosophie, mais il semble néanmoins que le parallèle dépasse la simple communauté idéologique. Quelques brefs exemples indiqueront une voie qui serait à développer. Parmi les théoriciens du roman, Käte Hamburger a montré que le roman faisait appel à un passé qui n'était pas chronologique. Le temps du passé perd dans la fiction sa valeur temporelle pour gagner la capacité à présenter un « autre » temps, un ailleurs qui ne serait pourtant pas irréel. Le roman, par son usage grammatical des temps, offre la possibilité de penser un autre monde. On pourrait imaginer que, chez Rancière, le refus de la temporalité historique emprunte implicitement à ces conceptions du temps de la fiction. Outre sa temporalité, la fiction narrative se caractérise par un usage particulier de la représentation des pensées ${ }^{8}$. Selon la linguiste Ann Banfield, le roman peut ainsi scinder une pensée et son expression?. Le contenu de la pensée est livré dans un langage qui n'est pas une parole du sujet de pensée. En d'autres termes, la subjectivation littéraire n'a pas la forme d'une parole prononcée. Il se produit alors une dissociation entre l'identité et le langage. La langue a la capacité de représenter mais laisse le sujet originaire dans l'opacité. Les «phrases sans parole » de Banfield ont ouvert la voie à un questionnement sur le sujet dans la langue. La désidentification du sujet littéraire n'estelle pas le modèle du sujet politique pensé par Rancière comme sujet en attente d'expression, à l'actualisation intermittente?

Aujourd'hui, la pensée linguistique a trouvé un nouvel essor philosophique dans une conception de la politique liée au pouvoir du groupe: les théories de l'énonciation servent à appuyer les revendica-

\footnotetext{
8. Dorrit Cohn, dans Transparent Minds, a ainsi montré que le roman donnait accès à l'intériorité des consciences.

9. Voir Phrases sans parole: Théorie du récit et du style indirect libre, Paris, Seuil 1995.
} 
tions au pouvoir de nouvelles communautés de parole. L'originalité de Rancière est de penser la parole collective sans passer par le groupe, ni, surtout, par un langage d'autorité. En utilisant le modèle de la subjectivation, il ancre la parole politique dans un fond subjectif sans sujet défini ${ }^{10}$. On retrouve alors l'élan commun à toute l'œuvre de Rancière: dès Le Maître ignorant il tentait de retracer la forme d'une pensée qui n'emprunterait pas un langage d'autorité pour se proférer. La polyphonie et la représentation narrative de la pensée offrent donc des modèles pour un sujet qui ne s'exprime que par la désidentification et dont l'identité première reste hypothétique.

Mais on peut alors retourner à Rancière les reproches qu'il adressait au sujet romantique. Dans La Parole muette, il reprenait la critique de Hegel à la fantaisie romantique ( $P M$, chap. 8). Le sujet infini serait le fantasme des romantiques préférant la duplicité à une incarnation réelle et donc limitée. On peut alors se demander pourquoi, dans le modèle de la subjectivation politique, le sujet se choisirait une désidentification limitée au lieu de l'infini des identités changeantes. Rancière ne donne pas la raison anthropologique qui arrêterait le choix d'un individu sur une figure d'altérité et le ferait finalement s'exprimer. Le paradigme romanesque s'articule mal avec la métaphore de la scène politique si présente chez lui. Parce qu'il n'est qu'une potentialité, le sujet risque de se perdre dans le tournoiement infini des masques, à la façon du neveu de Rameau ou des fantaisistes préromantiques, précurseurs du sujet moderne indéfini. L'ironie romantique représentait pour Hegel le danger d'une parole dont la responsabilité serait perdue. L'auteur y tient les contraires ensemble sans livrer d'intention définitive. Appliqué à la politique, ce modèle ouvre sur la situation inverse de l'acte de parole individuel rêvé par Rancière: une parole d'abstention, éternelle conversation, pur plaisir de la langue ou dégoût de la logorrhée infinie. Puisque le sujet est toujours présent dans la langue, pourquoi voter, et, surtout, comment avoir une opinion? La littérature doit peut-être ici être dissociée de la politique. À moins qu'elle n'en montre les limites.

10. Sandra Laugier au colloque La grammaire du Je proposait de lire chez Husserl un « sujet sans subjectivation » défini par le ton, rappel de la vocalité bakhtinienne. 


\section{Subjectivation politique et énonciation politque}

L'acte politique défini par Rancière laisse bien des questions irrésolues. Il s'agit tout d'abord de comprendre comment l'hypothèse de la subjectivation s'articule à des actes politiques. Si le passage du sujet à un groupe pose un problème, c'est aussi parce que l'historicité des formes politiques reste impensée. Rancière a choisi un modèle de parole qui est celui du roman $\mathrm{XIX}^{\mathrm{e}}$, et une figure, le prolétaire, dont les formes sont historiques. Il récuse la qualité politique des incarnations modernes de la parole collective, depuis les groupes culturels jusqu'à l'opinion mondiale, qui, selon lui, figent ou diluent le sujet.

Ensuite, le lien entre désidentification et revendication demeure problématique. Il semble alors que, chez lui, le modèle littéraire livre une dernière qualité de la subjectivation politique: elle est une hypothèse, c'est-à-dire un socle théorique mais aussi quelque chose qui est pensé hors du réel. Soit alors un autre modèle venu des théories linguistiques: le style indirect libre. Il se définit par trois éléments qui s'appliquent à la politique telle que Rancière la conçoit : polyphonie, utilisation des déictiques qui renvoient à la situation de l'hic et nunc de profération revendicatrice et, enfin, usage des temps du passé sans valeur chronologique. Le passé du style indirect libre s'apparente à ce passé du roman, dont on a vu le caractère atemporel. Ce passé hypothétique implique un saut hors de la temporalité quotidienne, et instaure l'ailleurs de la fiction. Or, c'est justement ce déplacement vers l'irréel qu'opère Rancière ${ }^{11}$. En prenant le modèle du roman, il fait aussi le choix de la fiction contre une pensée du monde réel.

Nombreuses sont les critiques, comme celles de Badiou, adressées à une réflexion qui détache les résultats des processus politiques qui les ont engendrés. Mais Rancière revendique ce détachement du plan des causes : pour lui, le pouvoir moderne de la fiction est d'avoir reconfiguré l'idée de causalité et de possibles, d'ouvrir une friction neuve entre les causes et les faits, l'imaginaire et le réel:

Au jeu métapolitique de l'apparence et de son démenti, la politique démocratique oppose cette pratique du comme si qui constitue les formes d'apparaître d'un sujet et qui ouvre une communauté esthétique $[\ldots] »(A B, 128)$.

11. Il écrit ainsi : «La littérature est le mode de discours qui défait les situations de partage entre la réalité et la fiction, le poétique et le prosaïque, le propre et l'impropre » $(A B, 189)$. 
Ce règne du «comme si », Rancière l'hérite autant de la philosophie de l'esprit que de la théorie de la fiction. Le Schein ou «als ob», « comme si », est à la fois l'apparaître messianique et l'envers présent en creux dans le monde, entre facticité et révélation. En ce sens, oui, la politique, c'est du roman.

Pour explorer le logos, la pensée de Rancière s'adosse au muthos sans penser ce socle fictionnel. Lacoue-Labarthe, dans Le Sujet de la philosophie, commentait l'idée nietzschéenne que la métaphysique prend la forme du roman. En partant de cette piste, on peut dire que Rancière n'utilise pas le roman pour satisfaire un désir refoulé de présence métaphysique, mais plutôt pour faire l'économie du récit, et livrer ainsi un sujet subjectivé hors de tout contexte et de toute temporalité.

Les limites de ce modèle romanesque sont à chercher dans son historicité. Le sujet politique dont parle Rancière est l'ouvrier, dont le nom a disparu. De même, la subjectivation qui l'accompagnait était celle du roman moderne: style indirect libre, monologue intérieur, jeu des points de vue, effacement du narrateur apparaissent avec le roman du XIX ${ }^{\mathrm{e}}$ siècle $^{12}$. Lorsque Rancière récuse les penseurs d'une « fin de la politique », on ne peut s'empêcher de penser qu'il refuse de reconnaître la fin de la politique telle qu'il l'a définie. Si l'on garde sa définition de « subjectivation avec revendication », il faudrait rechercher, en même temps que le nouveau nom qui remplacerait celui d'ouvrier, les techniques d'énonciation qui feraient entendre cette parole toujours inédite que doit être la politique. La conception du sujet a évolué en même temps que les formes contemporaines de l'art. Rancière a montré que la littérature était une forme de parole historiquement délimitée, il n'a pas pris en compte ce que cela impliquait pour sa pensée de la subjectivation, elle aussi relative à des formes d'énonciation historiques.

Quelle nouvelle littérarité offrira à cette subjectivation politique en souffrance sa voix? Quel art offrira la pensée du sujet politique revenu à la parole ? Penseur pourtant en éveil, pourtant à l'écoute des nouvelles formes d'art comme le cinéma, Rancière serait-il sourd aux nouvelles formes du sujet, restant un homme du XIX ${ }^{\mathrm{e}}$ siècle, ayant atteint la nouvelle frontière d'où il ne peut entendre quelle parole l'appelle au-delà?

12. Il est frappant qu'en s'intéressant au régime de représentation qu'est la mimesis comme fiction, Rancière ne se soit pas intéressé aux formes de représentation politiques (qu'il appelle la police) nées au XIX', en même temps que les formes littéraires de la subjectivation qu'il reprend. 